\title{
CONICAL LIMIT POINTS AND GROUPS OF DIVERGENCE TYPE
}

\author{
SUNGBOK HONG
}

\begin{abstract}
We use the Patterson-Sullivan measure to generalize Agard's theorem to all groups of divergence type. As a consequence, we prove that for a nonelementary group $\Gamma$ of divergence type, the conical limit set has positive Patterson-Sullivan measure.
\end{abstract}

\section{INTRODUCTION}

For a nonelementary group $\Gamma$ of hyperbolic motions of $B^{d+1}$, the critical exponent $\delta(\Gamma)$ is defined as

$$
\delta(\Gamma)=\inf \left\{\alpha: \sum_{\gamma \in \Gamma} e^{-\alpha(0, \gamma(0))}<\infty\right\}
$$

where $(0, \gamma(0))$ is the hyperbolic distance from 0 to $\gamma(0)$. The group $\Gamma$ is said to be of convergence or divergence type according as the series

$$
\sum_{\gamma \in \Gamma} e^{-\delta(\Gamma)(0, \gamma(0))}
$$

converges or diverges.

S. Agard [A, Theorem 1] showed that if $\delta(\Gamma)=d$ and $\Gamma$ diverges at the exponent $d$ then the $d$-dimensional Hausdorff measure of a certain subset of the limit set of $\Gamma$ is the same as the measure of $S^{d}$.

In this paper, we use the Patterson-Sullivan measure to generalize Agard's theorem to all groups of divergence type. The definition of the Patterson-Sullivan measure for groups of divergence type is reviewed in section 1. The PattersonSullivan measure can also be defined for groups of convergence type and the details for that case can be found in [N].

To state our result, we must define the shadow and the projection of a point $a \in B^{d+1}$. Denote by $B_{\rho}(a)$ the ball of center $a$ and non-Euclidean radius $\rho$ for $a \in B^{d+1}$ and $\rho>0$, and by $B_{\rho}$ the ball of radius $\rho$ at 0 . Given a point $a \in B^{d+1}, a \neq 0$, define the projection $\operatorname{pr}\{a\}$ in $S^{d}$ to be $a /|a|$ and the shadow $\operatorname{sh}\{a\}$ in $B^{d+1} \cup S^{d}$ to be the closed line segment $[a, \operatorname{pr}\{a\}]$. For a

Received by the editors April 28, 1993 and, in revised form, May 9, 1994.

1991 Mathematics Subject Classification. Primary 20H10, 30F35, 30F40.

Key words and phrases. Nonelementary group, group of divergence type Patterson-Sullivan measure, conical limit point.

Partially supported by a grant from GARC, KOSEF and BSRI-94-1422, Ministry of Education. 
set $E \subseteq B^{d+1}$ which does not contain 0, we define $\operatorname{pr} E=\bigcup_{x \in E} \operatorname{pr}\{x\}$ and $\operatorname{sh} E=\bigcup_{x \in E}[x, \operatorname{pr} x]$. For a set $E$ containing 0, we define $\operatorname{pr} E=S^{d}$.

Let $E_{\rho}(W) \subseteq S^{d}$ be defined by

$$
E_{\rho}(W)=\operatorname{pr}\left\{W T\left(B_{2 \rho}\right) \cap \operatorname{sh}\left\{W\left(B_{7 \rho}\right)\right\}\right\} .
$$

for fixed $T \in \Gamma$ and any $W \in \Gamma, \rho>0$. For some enumeration $W_{1}, W_{2}, \ldots$ of $\Gamma$, set

$$
\mathscr{E}_{\rho}(T)=\bigcap_{n=1}^{\infty} \bigcup_{k=n}^{\infty} E_{\rho}\left(W_{k}\right)
$$

Using Agard's approach together with properties of the Patterson-Sullivan measure $\mu_{0}$, we prove our main result Theorem 4.1.

Theorem 4.1. For every nonelementary discrete group $\Gamma$, there exists $\rho_{\Gamma}$ such that if there is a $T \in \Gamma$ with

(i) $T\left(B_{\rho_{\Gamma}}\right) \cap B_{\rho_{\Gamma}}=\varnothing$ and

(ii) $\mu_{0}\left[\mathscr{E}_{\rho_{\Gamma}}(T)\right]=0$,

then $\Gamma$ is of convergence type.

Theorem 4.1 implies the following result.

Corollary 4.2. If $\Gamma$ is of divergence type, then $\mu_{0}\left[\mathscr{E}_{\rho_{\Gamma}}(T)\right]>0$ for all but finitely many $T \in \Gamma$.

Define another subset of the limit set of $\Gamma$ as follows.

$\tilde{\mathscr{E}}_{\rho}(T)=\left\{q \in \Lambda ;\right.$ for every $p \in S^{d} \quad(p \neq q)$, there exists a sequence $\left\{W_{k}\right\} \subseteq$ $\Gamma$, with the geodesic with endpoints $p$ and $q$ meeting $W_{k}\left(B_{\rho}\right), W_{k} T\left(B_{\rho}\right)$ in order from $p$ to $q$, and $W_{k}\left(B_{\rho}\right) \rightarrow q$ as $k \rightarrow \infty$.

Making use of the above corollary, one can prove the following theorem.

Theorem 4.3. If $\Gamma$ is of divergence type, then $\mu_{0}\left[\tilde{\mathscr{E}}_{8 \rho_{\Gamma}}(T)\right]=\mu_{0}(\Lambda)$ for all but finitely many $T \in \Gamma$.

As an application, we have following results.

Corollary 5.1. Let $\Gamma$ be a nonelementary discrete group acting in $B^{d+1}$. If $\Gamma$ is of divergence type then the conical limit set $\Lambda_{\mathrm{c}}$ has positive Patterson-Sullivan measure.

Corollary 5.2. If $\Gamma$ is a nonelementary group of divergence type, then there exists $\rho_{\Gamma}$ such that $\mu_{0}\left[\mathscr{E}_{\rho_{\Gamma}}(T)\right]>0$ for all $T \in \Gamma$.

This paper is organized as follows. In section 1, we explain the PattersonSullivan measure and its properties which are needed in later sections. Most of these are from $[\mathrm{S}]$ and $[\mathrm{N}]$. In section 2, we examine some properties of shadows and projections. In section 3 , the connection between the measures $\mu_{x}$ and $\mu_{x^{\prime}}$ will be discussed. In section 4 , the main theorems are stated as Theorem 4.1 and Theorem 4.3. For the proof of these theorems, Sullivan's Shadow Lemma and Theorem 3.3 are indispensable. In section 5, we give applications of the main theorems. 
I would like to thank my mentor Darryl McCullough for his consistent encouragement and invaluable advice. The author wishes to thank the referee for invaluable suggestions.

\section{Preliminaries}

A conformal density of dimension $\alpha$ on a manifold $M$ is a function which assigns a positive measure $\mu(\rho)$ to each element $\rho$ in a nonempty collection of Riemannian metrics on $M$. It is assumed that if $\rho$ and $\rho^{\prime}$ are conformally the same, i.e, $\rho=\phi \rho^{\prime}$ where $\phi$ is a positive function, then $\mu=\mu(\rho)$ and $\mu^{\prime}=\mu\left(\rho^{\prime}\right)$ belong to the same measure class and the Radon-Nikodym ratio $d \mu / d \mu^{\prime}$ is $\phi^{\alpha}$. Thus

$$
\frac{d \mu}{d \mu^{\prime}}=\left(\frac{\rho}{\rho^{\prime}}\right)^{\alpha}
$$

We will employ a construction of Patterson-Sullivan to obtain a conformal density of a certain dimension $\alpha$ on the limit set $\Lambda$ of an arbitrary infinite discrete group $\Gamma$ of hyperbolic motions of $B^{d+1}$. This conformal density $\mu$ will be invariant under $\Gamma$ in the sense that $\gamma_{*} \mu(\rho)=\mu\left(\gamma_{\rho}\right)$, for all $\gamma$ in $\Gamma$ where $\gamma_{\rho}$ is the metric such that $\gamma$ is an isometry between $\rho$ and $\gamma_{\rho}$. For each $x$ in $B^{d+1}$, Patterson and Sullivan construct a measure $\mu_{x}$ by looking from $x$ at the orbit under $\Gamma$ of some point $y$.

A unit object at the point $\gamma y$ appears from $x$ to have size $e^{-(x, \gamma y)}$ where $(x, \gamma y)$ denotes the hyperbolic distance from $x$ to $\gamma y$. Thus in dimension $\alpha$ we want to associate the scale factor $e^{-\alpha(x, \gamma y)}$ to the point $\gamma y$. For each point $x$ in hyperbolic space we now show how to construct $\mu_{x}$. For $s$ a positive real number, consider the infinite series $g_{s}(x, y)=\sum_{\gamma \in \Gamma} e^{-s(x, y y)}$. For $x$ and $y$ fixed this series is proportional to

$$
\sum_{k=0}^{\infty} s_{k} e^{-k s}
$$

where $s_{k}$ is the number of orbit points in a half-open annulus of radii in $\left(k-\frac{1}{2}, k+\frac{1}{2}\right]$ centered about $x$. The series (1.1) converges for $s>\delta$ and diverges for $s<\delta$ where $\delta=\lim \sup _{k \rightarrow \infty} \frac{1}{k} \log s_{k}$. Since $\Gamma$ is discrete, $s_{k} \leq c e^{d k}$ for some constant $c$ depending on the minimal separation of the orbit points $\Gamma y$. Thus $\delta \leq d$.

If we define $n_{k}$ to be the number of orbit points in the closed ball of radius $k+\frac{1}{2}$ about $x$, then $n_{k}=\sum_{i=0}^{k} s_{i}$, so we may write $\delta=\lim \sup _{k \rightarrow \infty} \frac{1}{k} \log n_{k}$. Using the triangle inequalities $(x, \gamma y) \leq(x, y)+(y, \gamma y)$ and $(x, \gamma y) \geq$ $(y, \gamma y)-(x, y)$ yields

$$
e^{-s(x, y)} g_{s}(y, y) \leq g_{s}(x, y) \leq e^{s(x, y)} g_{s}(y, y) .
$$

In particular $\delta$ depends not on $x$ or $y$ but only on the discrete group $\Gamma$. We call $\delta$ the critical exponent and say that $\Gamma$ is of convergence or divergence type according as the series $\sum_{\gamma \in \Gamma} e^{-\delta(0, \gamma(0))}$ converges or diverges. Since we are interested in groups of divergence type at the critical value $\delta$, we will assume that $\lim _{s \rightarrow \delta} \sum_{\Gamma} e^{-s(x, y y)}=\infty$ for $s>\delta$, and this true for all $x, y$ using (1.2). Now we consider the family of measures $\mu_{s}(x)=\frac{1}{g_{s}(y, y)} \sum_{\Gamma} e^{-s(x, \gamma y)} D(\gamma y)$ where $D(\gamma y)$ is the unit Dirac mass at $\gamma y$. Appealing to Helly's theorem, 
there is a measure in the limit as $s_{i} \rightarrow \delta$, namely, $\mu_{x}=\lim _{s_{i} \rightarrow \delta} \mu_{s_{i}}(x)$. Since $g_{s}(y, y) \rightarrow \infty$ as $s \rightarrow \delta, \mu_{x}$ is concentrated on the cluster points of the orbit $\Gamma(y)$. Thus $\mu_{x}$ is a measure on the limit set $\Lambda(\Gamma)$.

Here are some facts which will be useful in later sections.

Proposition 1.1. There is a conformal density of dimension $\delta(\Gamma)$ supported on the limit set and invariant by $\Gamma$.

A proof of Proposition 1.1 can be found in Theorem 1 of [S].

Proposition 1.2. For a nonelementary discrete group $\Gamma$, the critical exponent $\delta(\Gamma)$ is positive.

Proof. See Corollary 1 in $\left.{ }_{\mathrm{i}} \mathrm{S}\right]$ and Corollary 3.4.5 in [N].

Proposition 1.3. If $\Gamma$ is a nonelementary discrete group which diverges at its critical exponent then the measures $\mu_{x}$ have no atomic part.

Proof. See Theorem 3.5.8 in [N].

Let $d_{0}$ be the metric on $S^{d}$ defined by $d_{0}(\xi, \eta)=\left|\cos ^{-1}(\xi \cdot \eta)\right|$. Then the $d_{0}$-radius $r_{\gamma}$ of $\operatorname{pr}\left\{\gamma\left(B_{\rho}\right)\right\} \subset S^{d}$ can be obtained from hyperbolic trigonometry (see Lemma 4.3.1 in [N]) as follows.

$$
\tan r_{\gamma}=\frac{\tanh \rho\left(1-|\gamma(0)|^{2}\right)}{2|\gamma(0)|} .
$$

Hence for $|\gamma(0)|$ close to 1 and sufficiently large $\rho$, we note from (1.3) that

$$
(1-|\gamma(0)|) \approx r_{\gamma} \text {. }
$$

The following result gives much useful information on the local structure of an invariant conformal density.

Proposition 1.4 (Sullivan's Shadow Lemma [N, Theorem 4.3.3]). Let $\Gamma$ be a discrete group acting in $B^{d+1}$ and $\mu$ a $\Gamma$-invariant conformal density of dimension $\alpha$ which is not a single atom. Then there exist constants $m, M$ such that, provided $\rho$ is large enough, for all except finite many $\gamma \in \Gamma$,

$$
m<\frac{\mu_{0}\left\{\gamma\left(B_{\rho}\right)\right\}}{r_{\gamma}^{\alpha}}<M .
$$

The above proposition is saying that near conical limit set, the measure class behaves somewhat like a Hausdorff $\alpha$-dimensional measure. Note that $\mu_{0}$ refers to picking a prescribed point in hyperbolic space.

Proposition 1.5. Let $\Gamma$ be a nonelementary discrete group acting in $B^{d+1}$ and $\mu$ a $\Gamma$-invariant conformal density of dimension $\alpha$. If $A$ is a $\Gamma$-invariant subset of the conical limit set, then either $\mu_{x}(A)=0$ or $\mu_{x}(A)=\mu_{x}\left(S^{d}\right)$.

Proof. See Theorem 4.4.4 in [N].

Now for $\gamma, \tau \in \Gamma$ and a conformal density $\mu$ of dimension $\alpha$ we have:

Lemma 1.6. For all sufficiently large $\rho$ and for each $t$, there exists a constant $K(\rho, t)$ so that if $(\gamma(0), \tau(0))<t$ then $\mu_{0}\left\{\gamma\left(B_{\rho}\right)\right\} / \mu_{0}\left\{\tau\left(B_{\rho}\right)\right\}<K(\rho, t)$.

Proof. By Proposition 1.4 we have $m<\mu_{0}\left\{\gamma\left(B_{\rho}\right)\right\} / r_{\gamma}^{\alpha}<M$ for almost all $\gamma$ in $\Gamma$. Note from $(1-|\gamma(0)|) / 2 \leq e^{-(0, \gamma(0))} \leq(1-|\gamma(0)|)$ and from (1.4) that there exists positive constants $k_{0}(\rho)$ and $K_{0}(\rho)$ such that for almost all $\gamma$,

$$
k_{0}(\rho) e^{-(0, \gamma(0))}<r_{\gamma}<K_{0}(\rho) e^{-(0, \gamma(0))} .
$$


Using this observation and Proposition 1.4, there exist positive constants $m_{0}$ and $M_{0}$ such that, provide that $\rho$ is large enough, for all except finite many $\gamma \in \Gamma$,

$$
m_{0}<\frac{\mu_{0}\left\{\gamma\left(B_{\rho}\right)\right\}}{e^{-\alpha(0, \gamma(0))}}<M_{0}
$$

Therefore

$$
\frac{\mu_{0}\left\{\gamma\left(B_{\rho}\right)\right\}}{\mu_{0}\left\{\tau\left(B_{\rho}\right)\right\}}<\frac{M_{0}}{m_{0}}\left(\frac{e^{-(0, \tau(0)}}{e^{-(0, \gamma(0)}}\right)^{\alpha} .
$$

So, it suffices to show the boundedness of $\left(e^{-(0, \tau(0))} / e^{-(0, \gamma(0))}\right)^{\alpha}$. But

$$
\frac{e^{-(0, \tau(0))}}{e^{-(0, \gamma(0))}}=e^{(0, \gamma(0))-(0, \tau(0))}<e^{t} .
$$

Hence $\mu_{0}\left\{\gamma\left(B_{\rho}\right)\right\} / \mu_{0}\left\{\tau\left(B_{\rho}\right)\right\}<K(\rho, t)$.

\section{SHADOWS AND PROJECTIONS}

From now on, we shall denote by $B_{\rho}(a)$ the ball of center $a$ and nonEuclidean radius $\rho$ for $a \in B^{d+1}$ and $\rho>0$. We denote by $B_{\rho}$ the ball of radius $\rho$ at 0 .

Given a point $a \in B^{d+1}, a \neq 0$, we define the projection $\operatorname{pr}\{a\}$ in $S^{d}$ to be $a /|a|$ and the shadow $\operatorname{sh}\{a\}$ in $B^{d+1} \cup S^{d}$ to be the closed line segment $[a, \operatorname{pr}\{a\}]$. For a set $E \subseteq B^{d+1}$ which does not contain 0 , we define $\operatorname{pr} E=$ $\bigcup_{x \in E} \operatorname{pr}\{x\}$ and $\operatorname{sh} E=\bigcup_{x \in E}[x, \operatorname{pr}\{x\}]$. For a set $E$ containing 0 , we define $\operatorname{pr} E=S^{d}$. Also we denote by $b(0: a, \rho)$ the projection of the hyperbolic ball of center $a$ and radius $\rho$ from the origin onto $S^{d}$. For later use, we require some simple relations among shadows and projections.

Proposition 2.1 [A, Lemma 3.1, 3.2, 3.3].

(i) If two geodesic rays with common terminal point $\xi \in S^{d}$ both meet a ball $B_{\rho}(a)$, and if one meets $B_{\sigma}(b)$ between $\xi$ and $B_{\rho}(a)$, then the other meets $B_{\sigma+2 \rho}(b)$. The estimate is sharpened to $B_{\sigma+\rho}(b)$ if one meets a itself.

(ii) $B_{\rho}(a) \subseteq \operatorname{sh}\left\{B_{2 \rho}(b)\right\}$ if any radius $r$ meets, in order, $0, b, B_{\rho}(a)$.

(iii) $B_{\rho+\tau}(a) \subseteq \operatorname{sh}\left\{B_{\sigma+\tau}(b)\right\}$ whenever $B_{\rho}(a) \subseteq \operatorname{sh}\left\{B_{\sigma}(b)\right\}$.

For $B_{\rho}(a)$ a ball in $B^{d+1}$, we consider the collection $\beta \subseteq B^{d+1} \cup S^{d}$ of all radial segments $[0, \operatorname{pr}\{x\}], x \in B_{\rho}(a)$. This set is called the solid angle supporting $B_{\rho}(a)$. Any image $\gamma(\beta)(\gamma \in \Gamma)$ will be a solid angle with vertex $\gamma(0)$. It is not hard to see geometrically that for all sufficiently large $\rho$, there is a positive function $\omega_{0}(\rho)$, such that

$$
\mu_{0}\left[\beta \cap S^{d}\right] \geq \omega_{0}(\rho)
$$

where $\beta$ is a solid angle containing $B_{\rho}$ (for more details, see the proof of Theorem 4.3.2[N]). From now on we only consider those elements $\left\{\gamma_{n}\right\} \subseteq \Gamma$ such that $\left(0, \gamma_{n}(0)\right)>\rho$. We need to define a set called a half-ball. By this we mean a set $\chi \subseteq B^{d+1} \cup S^{d}$, bounded by a Euclidean sphere orthogonal to $S^{d}$. We agree that $\chi$ includes its closure in $B^{d+1}$.

For the next three lemmas, we assume that the measure $\mu_{0}$ has no atomic part and $0<\mu_{0}(\Lambda)<\infty$. 


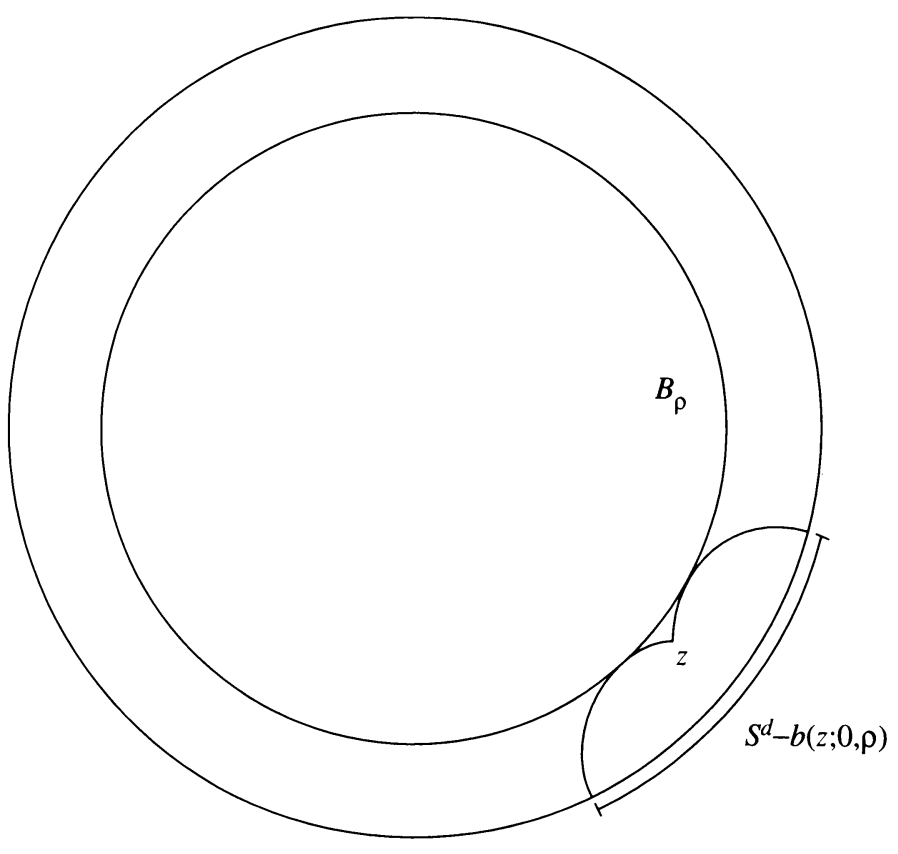

FIGURE 1

Lemma 2.2. Suppose that $\Gamma$ is a nonelementary discrete group. Then there exists $\rho_{0}$ sufficiently large such that for every $\rho \geq \rho_{0}$, for every $T \in \Gamma$ with $T\left(B_{\rho}\right) \cap B_{\rho}=\varnothing$, and for every half-ball $\chi$ such that $\chi \cap \Lambda \neq \varnothing$ and $\chi \cap B_{\rho}=\varnothing$, there exists $A \in \Gamma$, with $A\left(B_{\rho}\right), A T\left(B_{\rho}\right) \subseteq \chi$, and $A T\left(B_{\rho}\right) \subseteq \operatorname{sh}\left\{A\left(B_{2 \rho}\right)\right\}$.

Proof. Let $\lambda=\mu_{0}(\Lambda) / 3$. Then $\lambda>0$. Since $\mu_{0}$ has no atomic part, we may assume that there exists $\epsilon>0$ such that if $\Delta$ is a ball in $S^{d}$ of $d_{0}$-diameter at most $\epsilon$ then

$$
\mu_{0}(\Delta) \leq \lambda<\mu_{0}(\Lambda) .
$$

Choose $\rho_{0}$ so large that if $(z, 0)>\rho_{0}$. Then

$$
d_{0} \text {-diameter of } S^{d} \backslash b\left(z ; 0, \rho_{0}\right) \leq \frac{\epsilon}{5}
$$

where $b\left(z ; 0, \rho_{0}\right)$ is the projection of $B_{\rho_{0}}$ onto $S^{d}$ from $z$ as in Figure 1.

For $\rho \geq \rho_{0}$, let $\chi$ be a half-ball with $\chi \cap B_{\rho}=\varnothing$. For each $p \in \partial \chi \cap S^{d}$, we consider the half-ball $\chi\left(p, B_{\rho}\right)$ such that $\chi\left(p, B_{\rho}\right)$ is tangent to $\chi$ at $p$, to $B_{\rho}$ and $\chi\left(p, B_{\rho}\right) \cap \chi=\{p\}$. Note that from (ii),

$$
d_{0} \text {-diameter of }\left[\left\{\bigcup_{p \in \partial \chi \cap S^{d}} \chi\left(p, B_{\rho}\right) \cup \chi\right\} \cap S^{d}\right]<\frac{3 \epsilon}{5}<\epsilon \text {. }
$$

Now take a half-ball $\chi_{T}$ with $\chi_{T} \supset T\left(B_{\rho}\right)$ and $\chi_{T} \cap B_{\rho}=\varnothing$. Then from (i) and (iii) and reducing $\chi$ if necessary (in case $T\left(B_{\rho} \cap \chi \neq \varnothing\right)$, we can choose another half-ball $\chi^{\prime}$ with $\chi^{\prime} \cap T\left(B_{\rho}\right)=\varnothing$ but $\chi^{\prime} \cap \Lambda \neq \varnothing$ such that every ray from $T(0)$ to a point in $\chi^{\prime}$ meets $B_{\rho}$. Now by the Double Density Theorem [B], Theorem 5.3.8, we can choose a loxodromic element $A$ with fixed points $\xi \in \chi$ and $\eta \in \chi^{\prime}$ so that the axis of $A$ meets $B_{\rho}$ as in Figure 2. Taking 


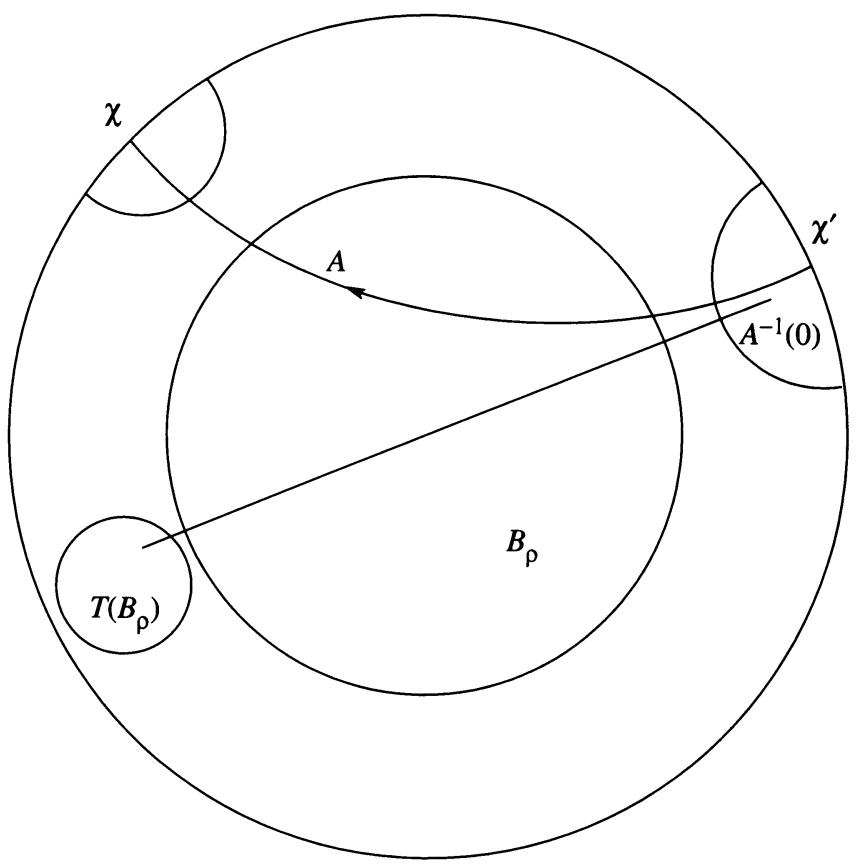

FIGURE 2

sufficiently high powers of $A$, we may assume that $A$ maps $B \backslash \chi^{\prime}$ inside $\chi$. This completes the proof.

Lemma 2.3. Given $\Gamma, T$ and $\rho \geq \rho_{0}$ as in Lemma 2.2, there exist a finite set of half-balls $\left\{\chi_{1}, \ldots, \chi_{n}: \chi_{i} \cap \Lambda \neq \varnothing\right\}$ and $\mathscr{A}=\left\{A_{1}, \ldots, A_{n}\right\} \subseteq \Gamma$, such that for any solid angle $\beta$ containing $B_{\rho}$, there exists $k$ with $\chi_{k} \subset \beta$, $A_{k}\left(B_{\rho}\right), A_{k} T\left(B_{\rho}\right) \subseteq \beta$, and $A_{k} T\left(B_{\rho}\right) \subseteq \operatorname{sh}\left\{A_{k}\left(B_{2 \rho}\right)\right\}$.

Proof. Choose a finite collection of round disks $\left\{D_{k}\right\}_{k=1}^{n}$ in $S^{d}$ so that each of which bounds a half-ball $\chi_{k}$ that does not meet the ball $B_{\rho}$ and $\bigcup_{k=1}^{n}$ Int $D_{k} \supset$ $\Lambda$.

Using (iii) in the proof of Lemma 2.2, for any half-ball $\chi$ with $\chi \cap \Lambda \neq \varnothing$ and $\chi \cap B_{\rho}=\varnothing$, we can find an open set $U(\chi)$ in $S^{d}$ as in Figure 3 (in case $d=1$ ) so that the part $G(\chi)$ between $B_{\rho}$ and $U(\chi)$ has the property that if a solid angle $\beta$ containing $B_{\rho}$ has a vertex in $G(\chi)$, then $\chi \subseteq \beta$ and $\bigcup_{i=1}^{n} U\left(\chi_{i}\right)=S^{d}$. Then if $\beta$ is any solid angle containing $B_{\rho}$, its vertex lies in some $G(\chi)$. For each $\chi_{k}$ with $\chi_{k} \cap \Lambda \neq \varnothing$, find $A_{k} \in \Gamma$ with the property of Lemma 2.2 .

Lemma 2.4 [A]. Given $\mathscr{A}$ as in Lemma 2.3, there exists a positive constant $M_{0}$ such that for any $\gamma \in \Gamma$, there exists $W=W(\gamma) \in \Gamma$, with the properties

(i) $W\left(B_{\rho}\right), W T\left(B_{\rho}\right) \in \operatorname{sh}\left\{\gamma\left(B_{\rho}\right)\right\}$,

(ii) $W T\left(B_{\rho}\right) \subseteq \operatorname{sh}\left\{W\left(B_{4 \rho}\right)\right\}$,

(iii) $\mu_{0}\left[\gamma\left(B_{\rho}\right)\right] \leq M_{0} \mu_{0}\left[W T\left(B_{\rho}\right)\right]$.

Proof. Given $\gamma \in \Gamma$, let $\beta$ be the solid angle supporting $\gamma\left(B_{\rho}\right)$. Then $\gamma^{-1}(\beta)$ is a solid angle containing $B_{\rho}$, and therefore containing $A\left(B_{\rho}\right)$ and $A T\left(B_{\rho}\right) \subseteq$ $\operatorname{sh}\left\{A\left(B_{2 \rho}\right)\right\}$, for some $A \in \mathscr{A}$. Define $W=\gamma A$. 


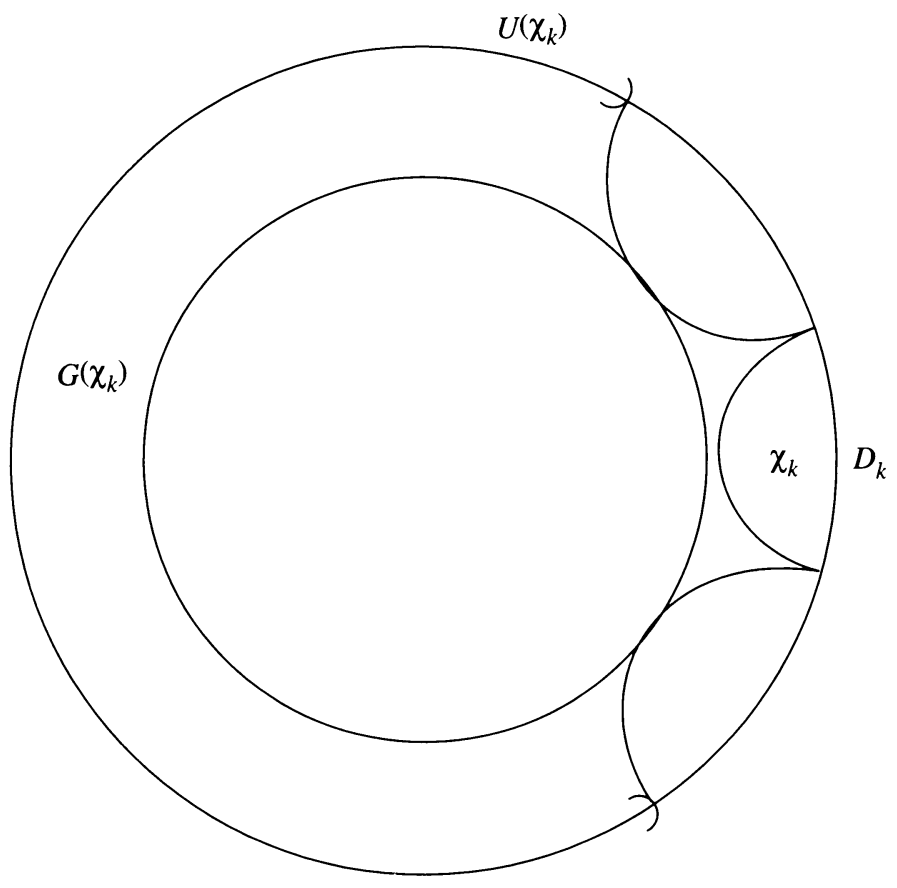

FIGURE 3

Part (i) is obvious from the construction. For (ii), take any radius $r=0 q$ meeting $W T$. From $\gamma^{-1}(q)$, draw the radius $s^{\prime}$ to 0 , and geodesic $r^{\prime}=$ $\gamma^{-1}(r)$. Set $s=\gamma\left(s^{\prime}\right)$. Now $r^{\prime}$ meets $A T\left(B_{\rho}\right)$ and $B_{\rho}$, and therefore $s^{\prime}$ meets $A T\left(B_{2 \rho}\right)$. Hence $s^{\prime}$ meets $A\left(B_{3 \rho}\right), s$ meets $W\left(B_{3 \rho}\right)$, and $r$ meets $W\left(B_{4 \rho}\right)$. Here Lemma 2.1 has been used. Part (iii) is evident from Lemma 1.6, once we calculate that

$$
\begin{aligned}
(W T(0), \gamma(0)) & =(A T(0), 0) \leq(A T(0), A(0))+(A(0), 0) \\
& =(T(0), 0)+(A(0), 0) \\
& \leq t_{0}=\max (A(0), 0)+(T(0), 0): A \in \mathscr{A} .
\end{aligned}
$$

Now we will characterize the conical limit set in terms of projections. First of all, we need the following result for the characterization.

Proposition 2.5 [N, Theorem 1.2.1]. Suppose $a \in B^{d+1}$ and $\xi, \eta \in S^{d}, \xi \neq \eta$. Let $s$ be the hyperbolic distance from $a$ to the geodesic joining $\xi$ and $\eta$. Then

$$
\cosh s=\frac{2|a-\xi||a-\eta|}{|\xi-\eta|\left(1-|a|^{2}\right)}
$$

Note that a point $\xi \in S^{d}$ belongs to $b(0: a, \rho)$ if and only if the radius to $\xi$ passes within a hyperbolic distance $\rho$ of $a$. By Proposition 2.5 , this is equivalent to

$$
|a-\xi||a+\xi|<\left(1-|a|^{2}\right) \cosh \rho
$$

With this result in hand, we next state the connection between conical limit set and projections. 
Proposition 2.6 [N, Theorem 2.4.5]. Let $\Gamma$ be a discrete group acting in $B^{d+1}$ and $\xi \in S_{d}$. Then $\xi$ is a conical limit point for $\Gamma$ if and only if for some $a \in$ $B^{d+1}$ and $\rho>0, \xi$ belongs to infinitely many projections $b(0: \gamma(a), \rho): \gamma \in \Gamma$.

\section{Derivatives}

Now we consider the connection between the measures $\mu_{x}$ and $\mu_{x^{\prime}}$. We will notice that it may be expressed in terms of the derivative of a Möbius transformation $\gamma$ with $\gamma(x)=x^{\prime}$. The derivative used in this connection is derived from the metric obtained on $S^{d}$ by radial projection. We begin with the great circle metric $d_{0}$ on $S^{d}$ defined by

$$
d_{0}(\xi, \eta)=\left|\cos ^{-1}(\xi \cdot \eta)\right| \text {. }
$$

Now for $x \in B$ choose a Möbius transformation $V$ preserving $B$ such that $V(x)=0$ and define

$$
d_{x}(\xi, \eta)=d_{0}(V(\xi), V(\eta)) .
$$

If $x \in B, \gamma$ is a Möbius transformation preserving $B$, and $\xi \in S^{d}$ we define

$$
\left|\gamma_{x}^{\prime}(\xi)\right|=\lim _{\eta \rightarrow \xi} \frac{d_{x}(\gamma(\xi), \gamma(\eta))}{d_{x}(\xi, \eta)}
$$

The next proposition gives the relation between the derivative and the ratio of Poisson kernels.

Proposition 3.1 [N, Lemma 3.4.2]. For any Möbius transformation $\gamma$ preserving $B$, for $x \in B$ and $\xi \in S^{d}$,

$$
\left|\gamma_{x}^{\prime}(\xi)\right|=\frac{P\left(\gamma^{-1}(x), \xi\right)}{P(x, \xi)}
$$

where $P$ denotes the Poisson kernel.

Proposition 3.2. There exists a positive function $M(\rho)$ such that, for all $\gamma \in \Gamma$, if $\xi_{1}, \xi_{2} \in S$ are confined to $\gamma^{-1}\left(\operatorname{pr}\left\{\gamma\left(B_{\rho}\right)\right\}\right)$, then

$$
\frac{\left|\gamma_{0}^{\prime}\left(\xi_{1}\right)\right|}{\left|\gamma_{0}^{\prime}\left(\xi_{2}\right)\right|} \leq M(\rho)
$$

Proof. If $\gamma(0)=0$ then put $M(\rho)=1$; otherwise note that

$$
\frac{\left|\gamma_{0}^{\prime}\left(\xi_{1}\right)\right|}{\left|\gamma_{0}^{\prime}\left(\xi_{2}\right)\right|}=\frac{P\left(\gamma^{-1}(0), \xi_{1}\right)}{P\left(\gamma^{-1}(0), \xi_{2}\right)}=\left(\frac{\left|\xi_{2}-\gamma^{-1}(0)\right|}{\left|\xi_{1}-\gamma^{-1}(0)\right|}\right)^{2} .
$$

Consider the projection of $\gamma^{-1}(0)$ to the boundary of $B_{\rho}(0)$ and denote by $x$ the point of projection onto $\partial B_{\rho}(0)$. Take the hyperplane $H$ which is tangent to $B_{\rho}(0)$ at $x$ and perpendicular to $S$. Let us denote by $S_{H}$ the sphere of intersection of $H$ with $S$. Then we have

$$
\left|\xi_{1}-\gamma^{-1}(0)\right|>\min _{\zeta \in S_{H}}\left\{\left|\zeta-\gamma^{-1}(0)\right|\right\},
$$

which shows the existence of $M(\rho)$.

Theorem 3.3. If $x \in B, \gamma$ is a Möbius transformation preserving $B^{d+1}$, and $E$ is a Borel subset of $S^{d}$ then

$$
\mu_{\gamma^{-1}(x)}(E)=\int_{E}\left|\gamma_{x}^{\prime}(\xi)\right|^{\delta} d \mu_{x}(\xi) .
$$


Proof. This is a consequence of Theorem 3.4.1 and Lemma 3.4.2 in [N].

Theorem 3.4. If $\Gamma$ is a discrete group preserving $B$ and if $\gamma \in \Gamma$, then for any $x \in B$

$$
\gamma^{*} \mu_{x}=\mu_{\gamma^{-1}(x)} .
$$

Proof. This is an immediate consequence of Theorem 3.2.4 in [N].

Remark. The last theorem, combined with Theorem 3.3, implies that for a Borel set $E$ of $S$ and $\gamma \in \Gamma$

$$
\mu_{x}(\gamma(E))=\int_{E}\left|\gamma_{x}^{\prime}(\xi)\right|^{\delta} d \mu_{x}(\xi)
$$

\section{MAIN THEOREMS}

For fixed $T \in \Gamma$ any $W \in \Gamma, \rho>0$, let $E_{\rho}(W) \subseteq S$ be defined by

$$
E_{\rho}(W)=\operatorname{pr}\left\{W T\left(B_{2 \rho}\right) \cap \operatorname{sh}\left\{W\left(B_{7 \rho}\right)\right\}\right\} .
$$

For some enumeration $W_{1}, W_{2}, \ldots$ of $\Gamma$, let

$$
\mathscr{E}_{\rho}(T)=\bigcap_{n=1}^{\infty} \bigcup_{k=n}^{\infty} E_{\rho}\left(W_{k}\right)
$$

The purpose of this section is to prove the following.

Theorem 4.1. For every nonelementary discrete group $\Gamma$, there exists $\rho_{\Gamma}$ such that if there exists $T \in \Gamma$ with

(i) $T\left(B_{\rho_{\Gamma}}\right) \cap B_{\rho_{\Gamma}}=\varnothing$ and

(ii) $\mu_{0}\left[\mathscr{E}_{\rho_{\Gamma}}(T)\right]=0$,

then $\Gamma$ is of convergence type.

Proof. If $\mu_{0}$ has an atomic part then by Proposition 1.3, $\Gamma$ is of convergence type. Therefore we assume that $\mu_{0}$ has no atomic part. Our object is to show that

$$
\sum_{\gamma \in \Gamma}(1-|\gamma(0)|)^{\delta}<\infty
$$

where $\delta$ is the critical exponent of $\Gamma$. Using Proposition 1.4 and (1.4), it is sufficient to show $\sum_{\gamma \in \Gamma} \mu_{0}\left[\gamma\left(B_{\rho_{\Gamma}}\right)\right]<\infty$. To do this, we take an enumeration $\gamma_{1}, \gamma_{2}, \ldots$ of $\Gamma$, fix $t>t_{0}$ where $t_{0}$ is chosen as in the proof of Lemma 2.4 and choose a new sequence $\gamma_{k_{0}}, \gamma_{k_{1}}, \gamma_{k_{2}}, \ldots$ with the property that $\gamma_{k_{0}}=\mathrm{id}$, and for $j \geq 1, k_{j}$ is the first index $k$ such that $\left(\gamma_{k}(0), \gamma_{k_{i}}(0)\right)>3 t$, for $i=0,1,2, \ldots, j-1$. The sets $\mathscr{F}_{j}=\left\{\gamma \in \Gamma:\left(\gamma(0), \gamma_{k_{j}}(0)\right) \leq 3 t\right\}$ are of constant cardinality $N(t)$, and $\bigcup \mathscr{F}_{j}=\Gamma$.

By Lemma 1.6, $\mu_{0}\left(B_{\gamma}\right) / \mu_{0}\left(B_{\gamma_{j}}\right)<K\left(\rho_{\Gamma}, 3 t\right)$ whenever $\gamma \in \mathscr{F}_{j}$. Hence

$$
\sum_{\gamma \in \Gamma} \mu_{0}\left(B_{\gamma}\right) \leq \sum_{j=0}^{\infty} \sum_{\gamma \in \mathscr{F}_{j}} \mu_{0}\left(B_{\gamma}\right) \leq \sum_{j=0}^{\infty} N(t) K\left(\rho_{\Gamma}, 3 t\right) \mu_{0}\left(B_{\gamma_{k_{j}}}\right)
$$

and so it suffices to prove $\sum_{j=0}^{\infty} \mu_{0}\left(B_{\gamma_{k_{j}}}\right)<\infty$. Now relabel this sequence $\gamma_{1}, \gamma_{2}, \ldots$, and set $\delta_{j}=W\left(\gamma_{j}\right) T$, where $W\left(\gamma_{j}\right)$ is as in Lemma 2.4. By 
Lemma 2.4, it suffices to show that

$$
\sum_{j=0}^{\infty} \mu_{0}\left[\delta_{j}\left(B_{\rho_{\mathrm{T}}}\right)\right]<\infty
$$

We note that the $\delta_{j}(0)$ are well spaced because

$$
\left(\delta_{j}(0), \delta_{i}(0)\right) \geq\left(\gamma_{j}(0), \gamma_{i}(0)\right)-\left(\gamma_{j}(0), \delta_{j}(0)\right)-\left(\gamma_{i}(0), \delta_{i}(0)\right)>3 t-2 t_{0} \geq t .
$$

Therefore we denote by $B_{j}$ the ball $\delta_{j}\left(B_{\rho_{\Gamma}}\right)$, and we shall assume that $t \geq 3 \rho_{\Gamma}$, so that these balls are disjoint.

We set up classes of balls:

$I_{0}=\left\{B_{\rho_{\Gamma}}\right\}$

$I_{1}=\left\{B_{k}: B_{k}\right.$ is not eclipsed by any $\left.B_{i}\right\}$

$$
I_{m}=\left\{B_{k}: B_{k} \text { is not eclipsed by any } B_{i} \notin \bigcup_{1 \leq j<m} I_{j}\right\} \text {. }
$$

In any class $I_{m}$, the shadows $\operatorname{sh}\left\{B_{k}\right\}$ are disjoint. Further, every $B_{j} \in I_{m+1}$ is eclipsed by some $B_{k} \in I_{m}$, for it were not, it would have been selected in an earlier class. Our main object is to show that

$$
\sum_{B_{j} \in I_{m+1}} \mu_{0}\left(B_{j}\right) \leq \frac{2}{3} \sum_{B_{k} \in I_{m}} \mu_{0}\left(B_{k}\right)
$$

Then we get

$$
\sum_{j=0}^{\infty} \mu_{0}\left(B_{j}\right)=\sum_{m=0}^{\infty} \sum_{B_{j} \in I_{m}} \mu_{0}\left(B_{j}\right) \leq \sum_{m=0}^{\infty}\left(\frac{2}{3}\right)^{m} \mu_{0}(\Lambda)<\infty .
$$

We shall further partition each class $I_{m+1}$ into two subclasses:

$I_{m+1}^{\prime}=\left\{B_{j} \in I_{m+1}: B_{j}\right.$ is partially eclipsed by some $\left.B_{k} \in I_{m}\right\}$,

$I_{m+1}^{\prime \prime}=\left\{B_{j} \in I_{m+1}: B_{j}\right.$ is totally eclipsed by some $\left.B_{k} \in I_{m}\right\}$.

We first consider the class $I_{m+1}^{\prime}$. Fix $B_{j} \in I_{m+1}^{\prime}$, partially eclipsed by $B_{k} \in I_{m}$. Then there exists $q$ in the boundary of $\operatorname{pr}\left\{B_{k}\right\}$ such that the radius from 0 to $q$ meets $\partial B_{k}$ at a point $b_{k}$ and $B_{j}$ at a point $b_{j}$ in the order $0, b_{k}, b_{j}$. Let us also denote $\delta_{j}(0)=a_{j}$ and $\delta_{k}(0)=a_{k}$. Putting $\rho=\rho_{\Gamma}$,

$$
\begin{aligned}
t & \leq\left(a_{k}, a_{j}\right) \leq\left(b_{k}, b_{j}\right)+2 \rho \\
& =\left(b_{j}, 0\right)-\left(b_{k}, 0\right)+2 \rho \\
& \leq\left(a_{j}, 0\right)-\left(a_{k}, 0\right)+4 \rho
\end{aligned}
$$

Hence from (1.5), we have

$$
\begin{gathered}
-\left(0, a_{j}\right) \leq-\left(0, a_{k}\right)+4 \rho-t, \quad e^{-\left(0, a_{j}\right)} \leq e^{-\left(0, a_{k}\right)+4 \rho-t}, \\
\frac{e^{-\left(0, a_{j}\right)}}{e^{-\left(0, a_{k}\right)}} \leq e^{4 \rho-t}, \quad \frac{r_{j}}{r_{k}} \leq \frac{K_{0}(\rho)}{k_{0}(\rho)} e^{4 \rho-t}, \quad r_{j} \leq \frac{K_{0}(\rho)}{k_{0}(\rho)}\left(e^{4 \rho}\right) e^{-t} r_{k},
\end{gathered}
$$

where $r_{j}$ is the $d_{0}$ radius of $\operatorname{pr}\left\{\delta_{j}\left(B_{\rho_{\Gamma}}\right\}\right)$ on $S^{d}$. This implies that the projections $\operatorname{pr}\left\{B_{j}\right\}, B_{j}$ partially eclipsed by $B_{k}$, all lie inside an "annular" region on $S^{d}$, of Euclidean inner radius asymptotic to $r_{\text {in }}=r_{k}\left(1-2 C(\rho) e^{-t}\right)$ and $r_{\text {out }}=r_{k}\left(1+2 C(\rho) e^{-t}\right)$ where $C(\rho)=\left\{K_{0}(\rho) / k_{0}(\rho)\right\} e^{4 \rho}$. 

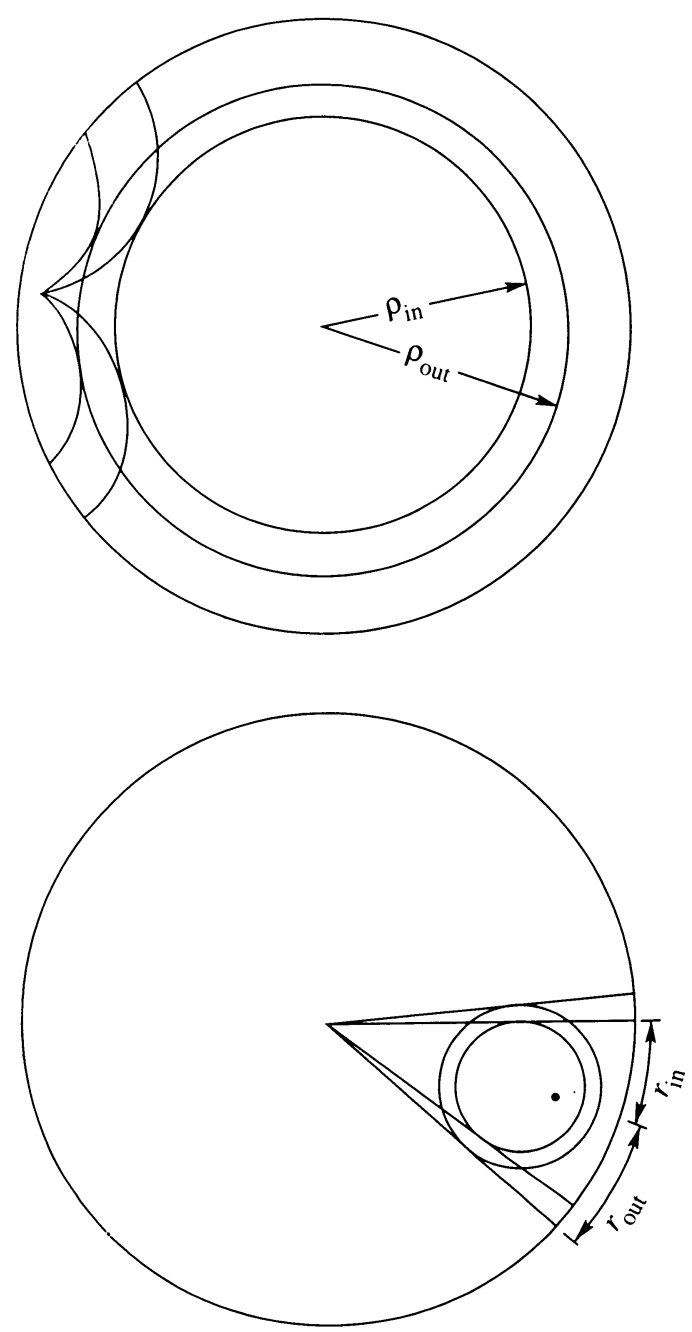

Figure 4

To complete the partially eclipsed case, we need the following lemma. Here we denote by $A\left(r_{\text {out }}, r_{\text {in }}\right)$ the annular region determined by $r_{\text {out }}$ and $r_{\text {in }}$ on $S^{d}$ as in Figure 4.

Lemma. $\mu_{0}\left[A\left(r_{\text {out }}, r_{\text {in }}\right)\right]<\frac{1}{3} \mu_{0}\left(B_{k}\right)$ for all $t$ sufficiently large relative to $\rho$.

Proof. Let $\rho_{\text {out }}$ be the hyperbolic radius of the ball centered at the origin such that the $d_{0}$-radius of $\delta_{k}\left(B_{\text {out }}\right)$ is $r_{\text {out }}$. Similarly we may define $\rho_{\text {in }}$. Hence we have

$$
A\left(r_{\text {out }}, r_{\text {in }}\right)=b\left(0: \delta_{k}(0), \rho_{\text {out }}\right)-b\left(0: \delta_{k}(0), \rho_{\text {in }}\right)
$$

Let

$$
\begin{aligned}
\lambda(t) & =\mu_{0}\left[b\left(\delta_{k}^{-1}(0): 0, \rho_{\text {out }}\right) \backslash b\left(\delta_{k}^{-1}(0): 0, \rho_{\text {in }}\right)\right] \\
& =\mu_{0}\left[\delta_{k}^{-1}\left\{b\left(0: \delta_{k}(0), \rho_{\text {out }}\right) \backslash b\left(0: \delta_{k}(0), \rho_{\text {in }}\right)\right\}\right] \\
& =\mu_{\delta_{k}(0)}\left[b\left(0: \delta_{k}(0), \rho_{\text {out }}\right) \backslash b\left(0: \delta_{k}(0), \rho_{\text {in }}\right)\right] .
\end{aligned}
$$


However, we have, from Theorem 3.3, Proposition 3.1, and (3.2) that

$$
\mu_{\delta_{k}(0)}\left[b\left(0: \delta_{k}(0), \rho_{\text {out }}\right) \backslash b\left(0: \delta_{k}(0), \rho_{\text {in }}\right)\right]=\int_{A\left(r_{\text {out }}, r_{\text {in }}\right)}\left[\frac{P\left(\delta_{k}(0), \xi\right)}{P(0, \xi)}\right]^{\delta} d \mu_{0}(\xi) .
$$

Now we approximate the Poisson kernel $P\left(\delta_{k}(0), \xi\right)$. Note that $P\left(\delta_{k}(0), \xi\right)=$ $\left(1-\left|\delta_{k}(0)\right|^{2}\right) /\left|\delta_{k}(0)-\xi\right|^{2}$.

To get the lower bound we note that if $\xi \in b\left(0: \delta_{k}(0), \rho_{\text {out }}\right)$ then from (1.4), we have

$$
\begin{aligned}
\left|\delta_{k}(0)-\xi\right| & \leq 1-\left|\delta_{k}(0)\right|+r_{\text {out }} \\
& =1-\left|\delta_{k}(0)\right|+r_{k}\left(1+2 C(\rho) e^{-t}\right) \\
& \approx 1-\left|\delta_{k}(0)\right|+\left(1-\left|\delta_{k}(0)\right|\right)\left(1+2 C(\rho) e^{-t}\right) .
\end{aligned}
$$

Since $\left(\delta_{k}(0), 0\right)>\rho$, we see that for a positive constant $a$, depending only on $\rho$,

$$
\left|\delta_{k}(0)-\xi\right| \leq\left(1+a\left(1+2 C(\rho) e^{-t}\right)\right)\left(1-\left|\delta_{k}(0)\right|\right) .
$$

Therefore

$$
\begin{aligned}
\int_{A\left(r_{\text {out }}, r_{\text {in }}\right)} P\left(\delta_{k}(0), \xi\right)^{\delta} d \mu_{0}(\xi) & \geq \frac{1}{\left(A+2 C(\rho) e^{-t}\right)^{2 \delta}\left(1-\left|\delta_{k}(0)\right|\right)^{\delta}} \int_{A\left(r_{\text {out }}, r_{\text {in }}\right)} d \mu_{0} \\
& =\frac{\mu_{0}\left[A\left(r_{\text {out }}, r_{\text {in }}\right)\right]}{\left(A+2 C_{0}(\rho) e^{-t}\right)^{2 \delta}\left(1-\left|\delta_{k}(0)\right|\right)^{\delta}} \\
& \geq \frac{c \mu_{0}\left[A\left(r_{\text {out }}, r_{\text {in }}\right)\right]}{\left(A+2 C_{0}(\rho) e^{-t}\right)^{2 \delta} r_{k}^{\delta}}
\end{aligned}
$$

where $A=a+1, C_{0}(\rho)=a C(\rho)$, and $c$ is a positive constant. But

$$
m<\frac{\mu_{0}\left\{\delta_{k}\left(B_{\rho}\right)\right\}}{r_{k}^{\delta}}<M
$$

therefore we have

$$
\mu_{0}\left[A\left(r_{\text {out }}, r_{\text {in }}\right)\right]<\frac{\lambda(t)}{c m}\left(A+2 C_{0}(\rho) e^{-t}\right)^{2 \delta} \mu_{0}\left(B_{k}\right) .
$$

Hence we conclude that for all $t$ sufficiently large relatve to $\rho$,

$$
\mu_{0}\left[A\left(r_{\text {out }}, r_{\text {in }}\right)\right]<\frac{1}{3} \mu_{0}\left(B_{k}\right) .
$$

Now we finish the argument of the partially eclipsed case by summing this over $B_{k} \in I_{m}$. Then we can conclude that for all sufficiently large $t$, we have

$$
\sum_{B_{j} \in I_{m+1}^{\prime}} \mu_{0}\left(B_{j}\right) \leq \frac{1}{3} \sum_{B_{k} \in I_{m}} \mu_{0}\left(B_{k}\right) .
$$

Now we consider the class $I_{m+1}^{\prime \prime}$. Fix $B_{k} \in I_{m}$, and let $J_{k}$ be the indices such that for $j \in J_{k}$, the balls $B_{j} \in I_{m+1}^{\prime \prime}$ are all totally eclipsed by $B_{k}$. Let $E_{k}=\operatorname{pr}\left\{\bigcup B_{j}: j \in J_{k}\right\}=\bigcup_{j \in J_{k}} \operatorname{pr}\left\{B_{j}\right\}$ and $F_{k}=\delta_{k}^{-1}\left(\operatorname{pr}\left\{B_{k}\right\}\right) \supseteq \delta_{k}^{-1}\left(E_{k}\right)$. 
Let $\beta$ be the solid angle supporting $B_{k}$, and consider $\delta_{k}^{-1}(\beta)$, a solid angle containing $B_{\rho}$. From (2.1), (3.1), and (3.2), we have

$$
\begin{aligned}
\omega_{0}(\rho) \mu_{0}\left[E_{k}\right] & \leq \mu_{0}\left[\delta_{k}^{-1}(\beta)\right] \int_{\delta_{k}^{-1}\left(E_{k}\right)}\left|\left(\delta_{k}\right)_{0}^{\prime}\right|^{\delta} d \mu_{0}(\xi) \\
& \leq \mu_{0}\left[\delta_{k}^{-1}\left(\operatorname{pr}\left\{B_{k}\right\}\right)\right] \max _{\xi \in F_{k}}\left|\left(\delta_{k}\right)_{0}^{\prime}\right|^{\delta} \mu_{0}\left[\delta_{k}^{-1}\left(E_{k}\right)\right] \\
& \leq \int_{F_{k}} d \mu_{0}(\xi) M(\rho) \min _{\xi \in F_{k}}\left|\left(\delta_{k}\right)_{0}^{\prime}\right|^{\delta} \mu_{0}\left[\delta_{k}^{-1}\left(E_{k}\right)\right] \\
& \leq \int_{F_{k}}\left|\left(\delta_{k}\right)_{0}^{\prime}(\xi)\right|^{\delta} d \mu_{0}(\xi) M(\rho) \mu_{0}\left[\delta_{k}^{-1}\left(E_{k}\right)\right] \\
& =\mu_{0}\left[\delta_{k}\left(F_{k}\right)\right] M(\rho) \mu_{0}\left[\delta_{k}^{-1}\left(E_{k}\right)\right] \\
& =\mu_{0}\left[B_{k}\right] M(\rho) \mu_{0}\left[\delta_{k}^{-1}\left(E_{k}\right)\right] .
\end{aligned}
$$

Hence we get

$$
\mu_{0}\left[E_{k}\right] \leq \frac{M(\rho)}{\omega_{0}(\rho)} \mu_{0}\left[B_{k}\right] \mu_{0}\left[\delta_{k}^{-1}\left(E_{k}\right)\right]
$$

From exactly the same argument as on p. 246 of [A] we have

$$
\delta_{k}^{-1} \delta_{j}\left(B_{\rho}\right) \subseteq \operatorname{sh}\left\{\delta_{k}^{-1}\left(W\left(B_{6 \rho}\right)\right)\right\}
$$

and

$$
\delta_{k}^{-1}\left(\operatorname{pr}\left\{B_{j}\right\}\right) \subseteq \operatorname{sh}\left\{\delta_{k}^{-1} \delta_{j}\left(B_{2 \rho}\right)\right\}
$$

where $\delta_{j}=W\left(\gamma_{j}\right) T$.

It follows that

$$
\delta_{k}^{-1} W T\left(B_{2 \rho}\right)=\delta_{k}^{-1} \delta_{j}\left(B_{2 \rho}\right) \subseteq \operatorname{sh}\left\{\delta_{k}^{-1} W\left(B_{7 \rho}\right)\right\}
$$

and therefore we get from (4.3) that

Observing that

$$
E_{\rho}\left[\delta_{k}^{-1} W\right]=\operatorname{pr}\left\{\delta_{k}^{-1} \delta_{j}\left(B_{2 \rho}\right)\right\} \supseteq \delta_{k}^{-1}\left(\operatorname{pr}\left\{B_{j}\right\}\right) .
$$

$$
\begin{aligned}
\left(\delta_{k}^{-1} W(0), 0\right) & =\left(W(0), \delta_{k}(0)\right)=\left(\delta_{j} T^{-1}(0), \delta_{k}(0)\right) \\
& \geq\left(\delta_{j}(0), \delta_{k}(0)\right)-\left(\delta_{j}(0), \delta_{j} T^{-1}(0)\right) \\
& \geq t-t_{0}
\end{aligned}
$$

we have

$$
\delta_{k}^{-1}\left(E_{k}\right)=\bigcup_{j \in J_{k}} \delta_{k}^{-1}\left(\operatorname{pr}\left\{B_{j}\right\}\right) \subseteq \bigcup E_{\rho}(A):(A(0), 0) \geq t-t_{0} .
$$

By taking Patterson-Sullivan measure, and using the hypothesis that

$$
\mu_{0}\left[\bigcap \bigcup E_{\rho}(A): A \in \Gamma\right]=0 \text {, }
$$

we see that

$$
\mu_{0}\left[\delta_{k}^{-1}\left(E_{k}\right)\right] \rightarrow 0 \quad(t \rightarrow \infty)
$$

and therefore we may select $t$ sufficiently large that

$$
\mu_{0}\left[\delta_{k}^{-1}\left(E_{k}\right)\right] \leq \frac{\omega_{0}(\rho)}{3 M(\rho)} .
$$


This shows that

$$
\sum_{B_{j} \in I_{m+1}^{\prime \prime}} \mu_{0}\left[B_{j}\right]=\sum_{B_{k} \in I_{m}} \mu_{0}\left[E_{k}\right] \leq \frac{1}{3} \sum_{B_{k} \in I_{m}} \mu_{0}\left[B_{k}\right],
$$

which along with (4.1), completes the proof of Theorem 4.1.

Corollary 4.2. If $\Gamma$ is of divergence type, then $\mu_{0}\left[\mathscr{E}_{\rho_{\Gamma}}(T)\right]>0$ for all but finitely many $T \in \Gamma$.

Define a subset of the limit set of $\Gamma$ as follows.

$\tilde{\mathscr{E}}_{\rho}(T)=\left\{q \in \Lambda\right.$ : for every $p \in S$, there exists a sequence $\left\{W_{k}\right\} \subseteq \Gamma$, with the geodesic with endpoints $p$ and $q$ meeting $W_{k}\left(B_{\rho}\right), W_{k} T\left(B_{\rho}\right)$ in order from $p$ to $q$, and $W_{k}\left(B_{\rho}\right) \rightarrow q$ as $\left.k \rightarrow \infty\right\}$.

Theorem 4.3. If $\Gamma$ diverges at its critical exponent, then $\mu_{0}\left[\widetilde{\mathscr{E}}_{8 \rho_{\Gamma}}(T)\right]=\mu_{0}(\Lambda)$ for all but finitely many $T \in \Gamma$.

Proof. By the definition of $\tilde{\mathscr{E}}_{\rho}(T)$ and Theorem $1(\mathrm{v})$ in [B-M], every point $q \in \widetilde{\mathscr{E}}_{\rho}(T)$ must be a conical limit point. Moreover $\widetilde{\mathscr{E}}_{\rho}(T)$ is $\Gamma$-invariant. To show this, let $q \in \tilde{\mathscr{E}}_{\rho}(T)$. For each $p \neq \gamma(q)$, namely $\gamma^{-1}(p) \neq q$, there exists a sequence $\left\{W_{k}\right\} \subset \Gamma$, with geodesic with endpoints $\gamma^{-1}$ and $q$ meeting $W_{k}\left(B_{\rho}\right), W_{k} T\left(B_{\rho}\right)$ in order from $\gamma^{-1}(p)$ to $q$ and $W_{k}\left(B_{\rho}\right) \rightarrow q$ as $k \rightarrow \infty$. Now we take the sequence $\left\{\gamma W_{k}\right\} \subseteq \Gamma$.

Since the set $\tilde{\mathscr{E}}_{\rho}(T)$ is a $\Gamma$-invariant, Proposition 1.5 shows that either $\mu_{0}\left(\widetilde{\mathscr{E}}_{\rho}(T)\right)=0$ or $\mu_{0}\left(\tilde{\mathscr{E}}_{\rho}(T)\right)=\mu_{0}(\Lambda)$. By definition, $q \in \mathscr{E}_{\rho_{\Gamma}}(T)$ if and

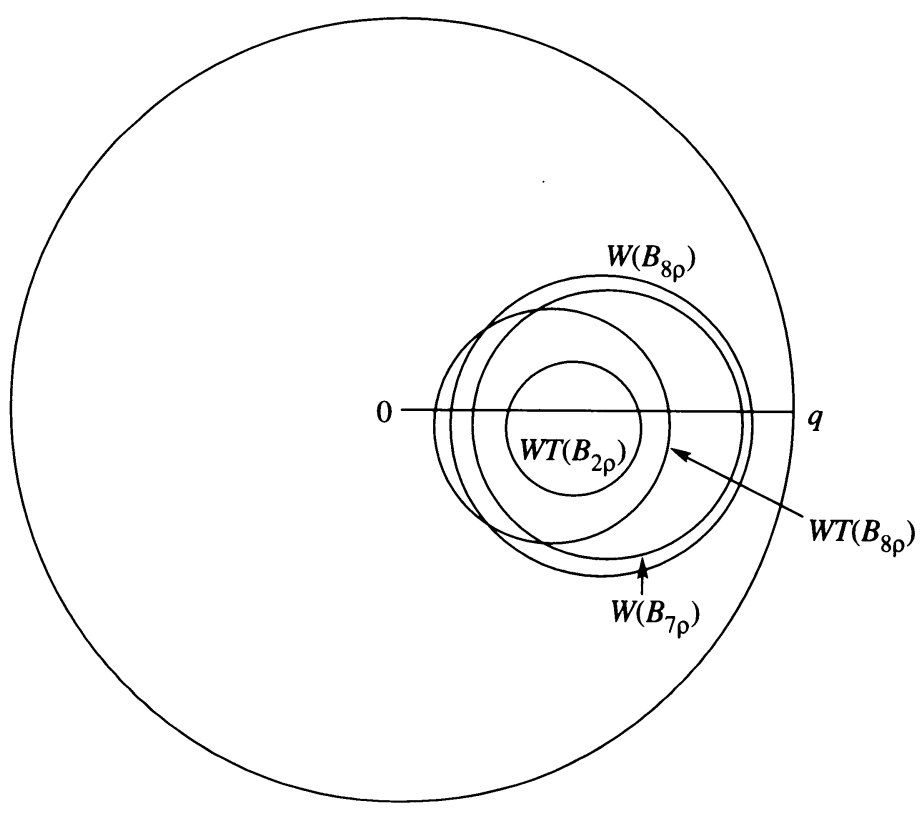

FiguRe 5 
only if $q$ lies in infinitely many of the sets $E_{\rho}\left(W_{k}\right)$. If this occurs then there exist infinitely many $W \in \Gamma$ with the radius from 0 to $q$ meeting in order 0 , $W\left(B_{7 \rho_{\Gamma}}\right), W T\left(B_{2 \rho_{\Gamma}}\right)$. This implies that any line from $p$ to $q$ meets, near $q$, infinitely many pairs $W\left(B_{8 \rho_{\Gamma}}\right), W T\left(B_{8 \rho_{\Gamma}}\right)$, and that $q \in \widetilde{\mathscr{E}}_{8 \rho_{\Gamma}}(T)$. In other words, $\mathscr{E}_{\rho}(T) \subseteq \widetilde{\mathscr{E}}_{8 \rho}(T)$ for all $T \in \Gamma$ such that $T\left(B_{2 \rho_{\Gamma}}\right) \cap B_{7 \rho_{\Gamma}}=\varnothing$. (In case $T\left(B_{2 \rho_{\Gamma}}\right) \cap B_{7 \rho_{\Gamma}}$ is nonempty, it is conceivable that we may have an example as in Figure 5. Namely, the radius from 0 to $q$ meets $0, W\left(B_{7 \rho_{\Gamma}}\right), W T\left(B_{2 \rho_{\Gamma}}\right)$ in that order but it meets in order $0, W T\left(B_{8 \rho_{\Gamma}}\right)$ and $W\left(B_{8 \rho_{\Gamma}}\right)$, failing to show that $q \in \widetilde{\mathscr{E}}_{8 \rho_{\Gamma}}(T)$.) Therefore $\mu_{0}\left[\widetilde{\mathscr{E}}_{8 \rho_{\Gamma}}(T)\right]=\mu_{0}(\Lambda)$ for all but finitely many $T \in \Gamma$.

\section{Applications}

Corollary 5.1. Let $\Gamma$ be a nonelementary discrete group acting in $B^{d+1}$. If $\Gamma$ is of divergence type then the conical limit set $\Lambda_{c}$ has positive Patterson-Sullivan measure.

Proof. Suppose for contradiction that $\mu_{0}\left(\Lambda_{c}\right)=0$. If $p \in \mathscr{E}_{\rho_{\mathrm{\Gamma}}}(T)$, then $p \in$ $E_{\rho_{\Gamma}}\left(W_{k}\right)$ for infinitely many $k$. Hence $p \in \operatorname{pr}\left\{W_{k} T\left(B_{2 \rho_{\Gamma}}\right)\right\}$ for infinitely many $k$. By Proposition 2.6, $p \in \Lambda_{c}$. This shows $\mathscr{E}_{\rho_{\Gamma}}(T) \subseteq \Lambda_{c}$ for every $T \in \Gamma$. Since $\mu_{0}\left(\Lambda_{c}\right)=0$, we have $\mu_{0}\left[\mathscr{E}_{\rho_{\Gamma}}(T)\right]=0$ for every $T \in \Gamma$. Then by Theorem $4.1, \Gamma$ is of convergence type. This is a contradiction.

Corollary 5.2. If $\Gamma$ is a nonelementary group of diverence type. Then there exists $\rho_{\Gamma}$ such that $\mu_{0}\left[\mathscr{E}_{\rho_{\Gamma}}(T)\right]>0$ for all $T \in \Gamma$.

Proof. If $T\left(B_{\rho_{\Gamma}}\right) \cap B_{\rho_{\Gamma}}=\varnothing$ then we are in the case of Theorem 4.1. Therefore we assume that $T\left(B_{\rho_{\Gamma}}\right) \cap B_{\rho_{\Gamma}} \neq \varnothing$. A point $\xi \in \mathscr{E}_{\rho_{\Gamma}}(T)$ if and only if $\xi \in E_{\rho_{\Gamma}}\left(W_{k}\right)$ for infinitely many $W_{k}$. Since $T\left(B_{\rho_{\Gamma}}\right) \cap B_{\rho_{\Gamma}} \neq \varnothing$, we have $W_{k} T\left(B_{2 \rho_{\Gamma}}\right) \subseteq W_{k}\left(B_{7 \rho_{\Gamma}}\right)$ for all $W_{k}$. Therefore $\xi \in \mathscr{E}_{\rho_{\Gamma}}(T)$ if and only if $\xi \in$ $\operatorname{pr}\left\{W_{k} T\left(B_{2 \rho_{\Gamma}}\right)\right\}$ for infinitely many $W_{k}$ if and only if there exists $k\left(2 \rho_{\Gamma}\right)>1$ such that

$$
\frac{\left|\xi-W_{k} T(0)\right|}{1-\left|W_{k} T(0)\right|} \leq k\left(2 \rho_{\Gamma}\right)
$$

Next we define, for $k \geq 1$, a point $\xi \in \Lambda_{c}(k)$ if there exists a sequence $\left\{\gamma_{n}\right\}$ such that

$$
\lim _{n \rightarrow \infty} \frac{\left|\xi-\gamma_{n}(0)\right|}{1-\left|\gamma_{n}(0)\right|} \leq k
$$

Then $\Lambda_{c}(k)$ is $\Gamma$-invariant and $\Lambda_{c}=\bigcup_{k=1}^{\infty} \Lambda_{c}(k)$. To show this, let $\xi \in \Lambda_{c}(k)$. Then there exists a sequence $\left\{\gamma_{n}\right\} \subset \Gamma$ such that

$$
\lim _{n \rightarrow \infty} \frac{\left|\xi-\gamma_{n}(0)\right|}{1-\left|\gamma_{n}(0)\right|} \leq k
$$

Applying $g \in \Gamma$, since $g$ is conformal and preserves $S^{d}$, we have

$$
\lim _{n \rightarrow \infty} \frac{\left|g(\xi)-g \gamma_{n}(0)\right|}{1-\left|g \gamma_{n}(0)\right|} \leq k
$$

Therefore $g(\xi) \in \Lambda_{c}(k)$. The second part is obvious.

Now assume that $\Gamma$ is of divergence type. Then $\mu_{0}\left(\Lambda_{c}\right)>0$ by Corollary 5.1. Using Proposition 1.5, for each $k \geq 1, \Lambda_{c}(k)$ has either 0 or full measure. Hence there exists $n_{0} \geq 1$ such that $\mu_{0}\left[\Lambda_{c}\left(n_{0}\right)\right]=\mu_{0}\left(\Lambda_{c}\right)$. 
For $\rho_{\Gamma}>0$ such that $k\left(2 \rho_{\Gamma}\right)>n_{0}$, we have $\mathscr{E}_{\rho_{\Gamma}}(T) \supset \Lambda_{c}\left(n_{0}\right)$. Therefore $\mu_{0}\left[\mathscr{E}_{\rho_{\mathrm{\Gamma}}}(T)\right]>0$. This completes the proof of Corollary 5.2.

\section{REFERENCES}

[A] S. Agard, A geometric proof of Mostow's rigidity theorem for groups of divergence type, Acta Math 151 (1983), 231-252.

[B] A.F. Beardon, The geometry of discrete groups, Springer-Verlag, New York, 1983.

[B-M] A.F. Beardon and B. Maskit, Limit points of Kleinian groups and finite sides fundamental polyhedra, Acta Math 132 (1974), 1-12.

[N] P.J. Nicholls, The ergodic theory of discrete groups, London Math. Soc Lecture Note Ser., vol. 143, Cambridge Univ. Press, London and New York, 1989.

[S] D. Sullivan, The density at infinity of a discrete group of hyperbolic motions, Inst. Hautes Etudes Sci. Publ. Math. 50 (1979), 171-202.

GaRC Department of Mathematics, Seoul National University, Seoul 151-742, Korea Current address: Department of Mathematics, Pusan Women's University, san 1-1, Kweobupdong, Buk-ku, Pusan, 616-060, Korea

E-mail address: shong@lotus.pwu.ac.kr 